\title{
LA TEORÍA DE MODELOS VISTA POR EL OJO DE DIOS*
}

Raúl Orayen

INSTITUTO de INVESTIGACIONES FilosófiCAS

UNAM

En el curso de diversos trabajos en los cuales discute temas conectados con la verdad y el realismo, Hilary Putnam siembra algunas dudas sobre la solución tarskiana clásica de las paradojas semánticas. Observa, con razón, que en la descripción misma de la jerarquía de lenguajes postulada para evitar las paradojas se usa un lenguaje que no se puede acomodar en dicha jerarquía. Para eliminar un serio problema, se adopta una relativización de la noción de verdad (a niveles del lenguaje); pero al explicar la solución se debe usar una suerte de lenguaje absoluto, como si se hablara desde una posición privilegiada que está fuera de la jerarquía y que usando una metáfora feliz Putnam describe como "el punto de vista del ojo de Dios". I Putnam parece estar de acuerdo con la opinión de Gödel según la cual, a diferencia de las paradojas semánticas, las de la teoría de conjuntos han quedado eficazmente resueltas. En este trabajo mostraré, sin embargo, que cuando la teoría de conjuntos se usa en la teoría de modelos actual, algunas aplicaciones de la misma dan lugar a una paradoja cuya solución más inmediata origina dificultades como las señaladas por Putnam en el caso de las paradojas semánticas. Creo que mi análisis de esta paradoja refuerza los puntos de vista de Putnam que están en el transfondo de sus observaciones sobre las paradojas semánticas, y creo también que ese análisis brinda apoyo adicional a cierto escepticismo que Putnam ha mostrado hacia la definición de verdad lógica en la línea de la teoría de modelos.

* Este texto es una traducción parcial y modificada de mi ponencia "A Paradox in the Semantics of Set Theory", presentada en el Simposio sobre la filosofia de Hilary Putnam (Taxco, Guerrero) en agosto de 1992. La investigación que originó la ponencia fue parte de mis actividades dentro del proyecto IN600889, del programa PAPIID de la DGAPA. Quiero expresar mi profundo agradecimiento al profesor Hilary Putnàm por sus útiles y alentadores comentarios al texto inglés; dicho texto, más largo y técnico que el presente artículo, será sometido para su publicación a una revista especializada en lógica, con pequeñas modificaciones y bajo el título original.

1 Su expresión es "God's cye view". 
En la primera sección de este artículo describiré la paradoja mencionada hacia el final del párrafo anterior. Mostraré luego que su solución más inmediata presenta las dificultades antes aludidas. En la sección final explicitaré más las conexiones de los tópicos analizados con las ideas de Putnam.

\section{La paradoja}

En lo que sigue, usaré la notación ' $T Q$ ' como abreviatura de la expresión "la teoría cuantificacional clásica de orden uno". TQ se desarrolla habitualmente con ayuda de una teoría formal, que consta de un lenguaje formalizado, un conjunto de axiomas y un conjunto de reglas de inferencia. ${ }^{2}$ Para mis propósitos no tiene importancia cuál sea la teoría elegida, supuesto que tenga las propiedades usuales de una formalización adecuada de esta parte de la lógica. Usaré, pues, ' $T Q$ ' como una suerte de "término singular ambiguo", que se referirá a cualquier sistema o teoría formal con los rasgos mencionados. La ambigüedad será inocua. De modo similar, ' $T C$ ' se usará para denotar ambiguamente cualquier sistema de teoría de conjuntos que esté formalizado dentro de $T Q$ y sea del tipo Zermelo-Fraenkel o del tipo von Neumann. ${ }^{3}$

Es importante aclarar que, tal como usamos nuestra simbología y terminología, $T Q$ es una teoría interpretada. Muchas de las afirmaciones que haremos serían falsas o carecerían de sentido si no presupusiéramos la semántica usual de $T Q$. Mencionaremos a menudo dos propiedades de $T Q$, su corrección y completitud. Estas son propiedades que posee el sistema formal que expresa $T Q$, respecto de su semántica habitual; si se adopta otra semántica para ese formalismo, podrían perderse los rasgos mencionados.

Un hecho curioso de los estudios lógicos contemporáneos es que TC se usa para desarrollar $T Q$ y viceversa. Examinemos esto con algún detalle.

$T C$ se utiliza en el estudio de $T Q$ en dos formas diferentes. En primer lugar, los conceptos básicos de $T C$ se usan para construir la semántica de $T Q$. Por ejemplo, una interpretación del lenguaje de $T Q$ se define como la elección de un conjunto no vacto $D$ (que será el dominio de las variables), la asignación a cada letra de predicado $n$-ádica de un conjunto de $n$-tuplas de elementos de $D$ y la asignación a cada constante individual de un elemento de $D .{ }^{4}$ Conjunto,

2 Por razones de simplicidad no tomaré en cuenta sistemas de deducción natural sin axiomas.

3 Si la ambigüedad de ' $T Q$ ' y ' $T C$ ' perturba al lector, puede pensar que estos símbolos denotan específicamente las formalizaciones de la teoría cuantificacional y la teoría de conjuntos que aparecen en los capítulos II y IV de Introduction to Mathematical Logic de Mendelson (3a. ed. Wadsworth, Inc., Estados Unidos, 1987). A menudo usaré este texto para ilustrar las cuestiones lógicas que me ocupan en el presente trabajo.

4 También por simplicidad no tomo en cuenta lenguajes con letras de función. 
conjunto no vacio, n-tupla y elemento son nociones técnicas de TC. El lógico define luego verdad bajo una interpretación y habitualmente utiliza para ello el concepto de secuencia denumerable, que también pertenece a $T C$. En segundo lugar, $T C$ se utiliza en el estudio de $T Q$ de modo más sofisticado cuando la primera teoría se usa esencialmente en la prueba de la corrección y completitud de la segunda.

Podría pensarse que en estas aplicaciones de los conceptos e ideas de teoría de conjuntos el lógico no necesita algo tan sofisticado como lo que he llamado $T C$ y podría usar simplemente una teoría de conjuntos intuitiva, no formalizada y no tan compleja como TC. Pero el lógico no desea esta simplificación peligrosa: conoce el riesgo de las paradojas que están al acecho entre las sencilleces de una teoría intuitiva de conjuntos y prefiere usar una teoría axiomática confiable. Habitualmente escoge una teoría formalizada en $T Q$ y del tipo Zermelo-Fraenkel o el estilo von Neumann. En otras palabras, usa lo que hemos llamado " $T C$ ". ${ }^{5}$ Otro posible malentendido es pensar que al usar $T C$ en el estudio de $T Q$, el lógico puede emplear la primera teoría como un sistema formal no interpretado. Pero éste no es el caso. Para que tenga sentido la aplicación de $T C$ en la construcción de la semántica de $T Q$, por ejemplo, se deben entender las nociones de conjunto, conjunto no vacio, etc., de la manera usual, y las explicaciones de los textos de lógica matemática elemental no dejan dudas al respecto. TC se entiende con su significado intuitivo habitual (presuponiéndose lo que en inglés se llama su intended model) en su aplicación a la semántica y metalógica de $T Q$.

El uso de $T Q$ en el desarrollo de $T C$ es más obvio. $T C$ está formalizada dentro de $T Q$ (recuérdese como definimos esa notación). Esto permite una precisión en la formulación de los axiomas de $T C$ que no era posible antes de la difusión de la lógica cuantificacional elemental. Por ejemplo, Zermelo formuló originalmente el llamado "axioma de separación" de una manera bastante oscura. El uso de la sintaxis de $T Q$ permitió la actual formulación, mucho más precisa y clara. Naturalmente, $T Q$ no se usa sólo para formular $T C$; los procedimientos deductivos de $T Q$ (sus verdades lógicas, sus reglas de inferencia) se emplean continuamente para extraer los teoremas de $T C$ y desarrollar la teoría. ${ }^{6}$

5 El uso de $T C$ en el estudio de $T Q$ origina a veces un problema expositivo en los libros de lógica matemática, porque el capítulo de $T Q$ suele estar antes que el de $T C$. Una solución de compromiso bastante socorrida es usar en el capítulo de $T Q$ únicamente ideas intuitivas de teoría de conjuntos (evitando así presuponer el conocimiento de una teoría que se explica después) pero teniendo la precaución de utilizar sólo herramientas de teoría de conjuntos que son legítimas desde el punto de vista de la (confiable) teoría axiomática que se introducirá luego (y se evita así el riesgo de las paradojas). Véase, por ejemplo, Mendelson, op. cit., p. 4, nota.

6 Otra vez, Mendelson, op. cit., suministra una ilustración apropiada. Véase su cap. IV. 
El hecho de que, como hemos comentado en los párrafos anteriores, TC se utiliza en el estudio de $T Q$ y viceversa, ha inquietado a veces por la posibilidad de que tal uso mutuo origine algún tipo de círculo vicioso. No me ocuparé de esta dificultad sino de una peor, relacionada con la inconsistencia. Para advertir cuál es el problema, tenemos que reparar en qué consiste formalizar una teoría intuitiva $T$ dentro de $T Q$. Supongamos que usted tiene algunas ideas acerca de algunos objetos, los objetos $O$. Usted piensa que los $O$ cumplen ciertas leyes generales. Piensa también que algunos $O$ en particular, a los que usted se refiere mediante ciertos términos singulares, poseen ciertas propiedades. Estas creencias suyas acerca de los $O$ conforman una teoría intuitiva $T$. Si usted ya le tomó el gusto a la matemática, la lógica y esas cosas (vicio relativamente inocuo), puede intentar una formalización de su teoría $T$ dentro de $T Q$. Necesitará entonces una teoría de orden uno $K$ que pueda expresar $T$. $K$ deberá poseer letras de predicado que puedan usarse para expresar las propiedades que usted atribuye a algunos $O$ a los cuales se refería mediante términos singulares. También necesitará tener en $K$ algunas constantes individuales que denoten los individuos a los que esos términos singulares se referian. $Y$ si usted quiere formalizar en $K$ esas leyes generales cuyo cumplimiento atribuía a los $O, K$ deberá tener fórmulas generales en las cuales se usen variables que puedan tener como dominio un conjunto que albergue a los $O$. Para transmitir brevemente la idea básica: si usted quiere formalizar $T$ dentro de $T Q$ deberá encontrar una teoría de orden uno $K$, tal que, bajo cierta interpretación de sus variables, letras de predicado y constantes individuales, $K$ diga de los objetos $O$ lo mismo que $T$. Parte de lo que se requiere entonces de $K$ para que sea una formalización adecuada de $T$, es que haya alguna interpretación de $K$ cuyo dominio sea un conjunto que albergue los objetos $O .^{7}$

La última observación del párrafo precedente servirá para hacer notar que la aplicación de $T C$ a $T Q$ y viceversa conduce a una paradoja. La dificultad surge cuando $T$ es una teoría de conjuntos del tipo Zermelo-Fraenkel o von Neumann, los $O$ son las entidades de que se ocupa $T$ y $K$ es una formalización de $T$ en $T Q$. Mostraré con algún detalle cómo surge la paradoja para el caso en que $T$ es del tipo Zermelo-Fraenkel. Luego será fácil extender el resultado a una teoría del estilo von Neumann. En lo que sigue, entonces, $T$ es una teoría del tipo Zermelo-Fraenkel, los $O$ son los objetos de que se ocupa ${ }^{8}$ y $K$

7 En los casos típicos ese conjunto será simplemente el conjunto de los $O$ : tendrá como elementos a todos los $O$ y sólo a ellos. Pero para todas las cuestiones discutidas en el texto nada cambia si se escoge como dominio un conjunto que contiene como miembros a todos los $O$ y algunas cosas más.

8 Supondremos que todos esos objetos son conjuntos y que no hay referencia alguna a urelementos. No tenemos necesidad de suponer que los conjuntos de que se ocupa una teoría de este tipo son todos los conjuntos. Puede sostenerse que, en algún sentido, el universo de conjuntos 
es una formalización de orden uno de $T$. Surge una paradoja cuando el lógico establece las dos conexiones entre $T Q$ y $T$ que se detallan a continuación y acepta dos supuestos sobre $T$ que formulamos luego.

1) La semántica de $T Q$ se construye con $T$. En particular, se adopta la restricción de que sólo conjuntos aceptados por $T$ pueden usarse en tal semántica: si en la semántica se usa el conjunto $A, T$ debe afirmar que entre las entidades de que se ocupa se encuentra $A$. En consecuencia, no se le puede asignar a una teoría de orden uno $K$, una interpretación cuyo dominio no sea provisto por $T$ en el sentido ya aclarado.

2) $T$ puede formalizarse dentro de $T Q$. En otras palabras, hay una teoría de orden uno $K$ que puede expresar $T$.

1) y 2) sintetizan las conexiones que describimos antes entre $T Q$ y una teoría de conjuntos del tipo Zermelo-Fraenkel o del tipo von Neumann, sólo que para comenzar nuestro análisis tomamos una teoría del primer tipo. ${ }^{9}$ Recordemos que ante el riesgo de las paradojas el lógico construye la semántica de $T Q$ restringiéndose a los recursos de una teoría axiomática confiable, usualmente de los tipos considerados; 1) establece ese hecho, bajo el supuesto de que la teoría elegida es del primer tipo mencionado (se analizará luego el otro caso); 2) recuerda que el lógico considera que la teoría de conjuntos elegida es formalizable en el orden uno. Pero si $T$ es del estilo Zermelo-Fraenkel, el lógico sabe que:

3) $T$ afirma que, entre todos los objetos de que se ocupa, no hay ninguno que contenga como elementos a todos esos objetos. Usando la simbología anterior: $T$ afirma que entre los $O$ no hay ninguno que contenga como elementos a todos los $O$. Con terminología más usual, teniendo en cuenta que los $O$, los objetos de que habla $T$, son conjuntos: $T$ afirma que entre todos los conjuntos de que habla no bay ninguno que contenga como elementos a todos esos conjuntos. ${ }^{10}$

de Zermelo-Fraenkel no agota el reino de las entidades que pueden llamarse propiamente conjuntos. La paradoja se deriva igual, se acepte o no esta tesis. Sin embargo, la cuestión ontológica que está en juego es relevante para juzgar si ciertas soluciones de la paradoja son filosóficamente aceptables.

9 En la argumentación siguiente, $T$ no es, estrictamente hablando, un caso de $T C$, porque no se supone que esté formalizada dentro de $T Q$; es más bien una teoría de conjuntos no completamente formalizada todavía y que contiene las ideas y principios básicos de una teoría del tipo $Z F$ (la que se expone en el clásico Abstract Set Theory, de Fraenkel, es un buen ejemplo). Los problemas que conducen a la paradoja se perciben más vívidamente en el caso de una teoría así. Aplicar luego la argumentación a TC es trivial.

10 Esta afirmación de la teoría Zermelo-Fraenkel se expresa habitualmente como la negación de la existencia de un conjunto universal. Pero el adjetivo 'universal' puede confundir; no se usa aqui en lo que podría llamarse su sentido "absoluto". Lo que niega la teoría es que exista algo 
Por otra parte, el lógico no lo puede probar, pero cree que:

\section{4) $T$ es consistente.}

Se llega a una perturbadora contradicción de la manera siguiente. De acuerdo con 2), $T$ se puede formalizar en el orden uno, i.e., hay una teoría de orden uno $K$ que puede expresar $T$. Pero de acuerdo con lo que habíamos observado oportunamente, si $K$ puede formalizar $T$, hay una interpretación de $K$ cuyo dominio es un conjunto que tiene como miembros a todos los objetos de que habla $T$. Pero de acuerdo con 1), la semántica del orden uno está restringida a los recursos que pueda proporcionar $T$ misma: no puede haber una interpretación de una teoría de orden uno $K$ cuyo dominio de interpretación no sea un conjunto provisto por $T$. Pero, si: a) realmente existe la teoría $K$ que expresa $T$, y para ello, b) debe existir un conjunto, admisible como dominio de interpretación, que tiene como miembros todos los objetos de que habla $T$, y además, c) todo conjunto que se use en la semántica está provisto por $T$ mismo, llegamos a que: $T$ acepta la existencia, entre las entidades de que se ocupa, de un conjunto que tiene como miembros todos los objetos de que habla $T$. Pero de acuerdo con 3 ), $T$ afirma que no existe un conjunto tal entre las cosas de las que habla (en terminología técnica, $T$ afirma que entre los valores de sus variables no hay uno que contenga todos esos valores). Y como, por 4), $T$ es consistente, no acepta la existencia de un conjunto que tiene como miembros todos los objetos de que habla $T .^{11}$

La paradoja surge, fundamentalmente, porque en la semántica de $T Q$ se adopta la restricción de que sólo se usarán recursos de cierta teoría de conjuntos dada para construir las interpretaciones de teorías de orden uno y al mismo tiempo se piensa que la teoría de conjuntos en cuestión puede formalizarse como una teoría tal; pero para que tal formalización fuera posible, la teoría debe brindar recursos de que carece, si es consistente. Es fácil notar que si la teoría de conjuntos intuitiva que se quisiera formalizar fuera del tipo von

dentro del universo de la teoría que contenga todas las entidades de ese universo, y no niega la existencia dentro del mismo de algo que contenga todo (aunque la segunda negativa se desprende de la primera, no significan lo mismo; más aun, si contener las entidades $A$ significa contener a las $A$ y sólo a ellas, para desprender la segunda negativa de la primera se requiere un axioma de separación).

11 Podría pensarse que ya se había llegado a una inconsistencia con la oración anterior. No es así. En el texto estamos haciendo afirmaciones de un carácter metalógico, hablando de teorías formales y sus relaciones. Si $T$ fuera inconsistente, podría ser cierto que $T$ afirmara que no existe el conjunto de los $O$ y ser cierto también que aceptara (afirmara) la existencia del conjunto de los $O$. En un ejemplo análogo pero más sencillo: puede ser cierto que Juan afirmó que no existe la entidad $B$ y que Juan afirmó que existe $B$, porque Juan puede contradecirse. La afirmación metalógica de que una teoría formal contiene afirmaciones contradictorias, no es contradictoria y puede ser cierta (en cuyo caso la teoría formal, y no la afirmación metalógica acerca de ella, es inconsistente). Se necesita, entonces, el supuesto 4) para arribar a nuestra paradoja. 
Neumann, surgirían dificultades similares. Una teoría tal puede suministrar los recursos para la semántica de una teoría del estilo Zermelo-Fraenkel; pero tampoco puede dar los elementos que permitan su propia formalización. Ejemplifiquemos con la teoría $N B G$ del capítulo IV de Mendelson (op. cit.). $N B G$ posee, aparentemente, una clase universal; pero en realidad tal clase ${ }^{12}$ no tiene como miembros a todos los objetos de que habla la teoría. En realidad, ninguna de las clases de $N B G$ alberga todos las entidades de las que ésta se ocupa: ninguna tiene como miembro la clase universal, por ejemplo. Se sigue de esto que tampoco $N B G$ puede suministrar el dominio que se necesita para dar una interpretación de una teoría de orden uno que la exprese.

La observación final de esta sección será un tanto insólita. La misma paradoja que he mostrado indica que la definición que di originalmente de ' $T C$ ' no le asigna ninguna denotación a ese supuesto "término singular ambiguo". La razón es que, como muestra la paradoja, una teoría de conjuntos del tipo Zermelo-Fraenkel o von Neumann no puede realmente ser formalizada dentro de $T Q$. La definición debe alterarse, cambiando el requisito de formalización dentro de $T Q$ por uno más superficial: la teoría en cuestión debe ser susceptible de "escribirse" en fórmulas de $T Q$ que la expresen cuando se da a los signos de $T Q$ un sentido intuitivo (que no corresponde necesariamente a ninguna de las interpretaciones de la semántica "oficial"). ${ }^{13}$

\section{Una jerarquía de conjuntos}

En mi formulación de las premisas que conducen a la paradoja, hice hincapié en un hecho acerca de las teorías del tipo Zermelo-Fraenkel que ahora tendrá suma relevancia. Una teoría de este tipo - que, usando nuevamente un "término singular ambiguo", llamaré en adelante ' $Z F$ '- hace afirmaciones generales acerca de una familia de entidades de las cuales se ocupa (cuando la teoría está "escrita" en lenguaje cuantificacional puede interpretarse que esas

12 'Clase' no es sinónimo de 'conjunto' en este sistema. Es un término más amplio, que abarca tanto colecciones que pertenecen a otras colecciones (los conjuntos) como colecciones que no pertenecen a ninguna colección (las clases propias).

13 Supongo que la mayoría de los lógicos saben que algo raro ocurre con la semántica de una teoría de conjuntos típica supuestamente formalizada en el orden uno. Sin embargo, hasta donde mis amigos lógicos y yo sabemos, nadie mostró explícitamente que se llega a una contradicción formal a partir de ciertos supuestos comunes sobre la semántica de $T Q$ y $T C$ (pero en el momento de escribir estas líneas no he localizado todavía una nota de Kreisel que me mencionó Putnam y de la cual piensa él que constiruyó la primera alusión al problema -aunque en la ocasión no tuviera presente la referencia exacta). Alchourrón me atribuye el hallazgo de la dificultad (que él llama "Orayen's paradox") en su "On The Philosophical Adequacy of Set Theories" (Theoria, 2a. época, año II, pp. 567-574). Quine me dice en una nota que no conocia la paradoja antes de una carta con ni primera formulación del problema y se muestra de acuerdo con la solución que entonces sugería (la solución I de la ponencia "A Paradox in the Semantics of Ser Theory"). 
entidades son todos los valores de sus variables). Llamemos conjuntos $Z$ a todas las entidades de las que habla $Z F$. $Z F$ sólo hace afirmaciones generales acerca de los conjuntos $Z$ (en general, las generalizaciones de una teoría sólo dicen cosas acerca de los valores de sus variables). $Z F$ no dice que no hay ninguna entidad (colección, clase, o lo que sea) que no contenga todos los conjuntos $Z$ como elementos. Lo que afirma explícitamente es que entre los conjuntos $Z$ (los valores de sus variables, las entidades de las que habla) no hay uno que contenga todos los conjuntos $Z$ como elementos. Pero cuando esto queda claro surge la posibilidad de construir una extensión de $Z F$, con un dominio de valores ampliado, entre cuyas nuevas entidades se cuenta una que contiene como elementos todas las entidades de que se ocupa $Z F$, todos los conjuntos $Z$. Esto no contradice a $Z F$ porque $Z F$ no dice que no haya ninguna entidad que no contenga a los conjuntos $Z$, decía que no había tal cosa entre los conjuntos $Z$.

Las consideraciones del párrafo anterior pueden formalizarse. Partiendo de $Z F$ se puede construir una extensión en la que se postule una entidad que contenga como elementos a todos los conjuntos $Z$ (una obvia prueba por el absurdo demostrará que no es un conjunto $Z$ ). La nueva entidad puede identificarse con el dominio de $Z F$ en su interpretación intuitiva habitual. La teoría extendida puede ir más lejos: introduciendo una letra de predicado con el significado de 'es un conjunto $Z$ ', se pueden postular infinidad de colecciones de conjuntos $Z$ que no se encontraban entre las entidades de las que se ocupaba $Z F$. Con el dominio ampliado (los conjuntos $Z$ más las nuevas colecciones) se puede tener todo lo requerido para construir nuevas interpretaciones de las teorías de orden uno, entre ellas la interpretación que no teníamos antes: una interpretación bajo la cual una teoría de orden uno $K$ puede expresar lo que dice $Z F$ en su sentido intuitivo habitual. Naturalmente, surge el temor de que tan temeraria ampliación acarree inconsistencias. Pero el temor puede disiparse. De manera similar a metateoremas que muestran que el pasaje de una teoría del tipo Zermelo-Fraenkel a una del estilo von Neumann no incrementa el riesgo de contradicciones, se puede construir una prueba de consistencia relativa que nos asegure la consistencia de la teoría extendida si la $Z F$ original era consistente. ${ }^{14}$

14 En la sección 3 del trabajo antes citado, "A Paradox in The Semantics of Set Theory", se hace una exposición formal minuciosa y detallada de estos temas. En particular, se describe la construcción de la extensión de $Z F$, se muestra que tal extensión proporciona un modelo del formalismo de $Z F$ acorde con su sentido intuitivo y se detalla la prueba de consistencia relativa que, si bien usa recursos semejantes a la prueba análoga respecto de $Z F$ y $N B G$, es conceptualmente muy distinta de ella. El objetivo del presente artículo no es dar el detalle formal de estas cuestiones lógicas sino mostrar algunas consecuencias de tipo filosófico que muestran un paralelo con ideas de Purnam sobre las paradojas semánticas. 
Los dos párrafos anteriores sugieren una solución de la paradoja que resulta bastante natural a quienes están familiarizados con las herramientas de la teoría de conjuntos. La solución exige abandonar la idea de que una teoría de conjuntos puede suministrar todas las colecciones de entidades que se requieren para todas las interpretaciones del lenguaje de $T Q$. Se introduciría una extensión de $Z F$ para proporcionar las colecciones que se necesitan en una interpretación que permita expresar esa teoría. Es fácil mostrar que la nueva teoría tampoco puede brindar lo que se necesita para su semántica. Se requiere otra teoría para lograr la tarea, y el problema y su solución se reiteran indefinidamente. Se obtiene así una jerarquía infinita de teorías y una jerarquía infinita de conjuntos. Ninguna teoría de conjuntos de la serie puede proporcionar su propia semántica, pero para cada una de ellas hay otra que puede hacerlo. Renunciamos a una concepción simplista según la cual hay una TC con la cual se puede construir la semántica de $T Q$ y abandonamos entonces los supuestos sobre $T Q$ y $T C$ que condujeron a consecuencias paradójicas.

Pero no todo queda resuelto a pesar de las apariencias. Cuando se estudia $T Q$ se define verdad logica como verdad bajo toda interpretación. Ahora tenemos una variedad infinita de interpretaciones. Si deseamos una noción de verdad lógica aplicable a cualquier teoría que se exprese con el formalismo de $T Q$, podemos conservar la letra de la definición usual pero pensando ahora que 'toda interpretación' se debe referir a las interpretaciones de todos los niveles de la jerarquía de conjuntos descripta. Pero entonces, al definir verdad lógica estamos cuantificando sobre todas las interpretaciones y en consecuencia sobre todos los conjuntos, de todas las jerarquias. Me temo que otra vez estamos contemplando algo desde el punto de vista del ojo de Dios. Se trata ahora de la semántica generalizada de $T Q$, o la teoría de modelos clásica. Para que se vea claramente qué dificultades enfrentamos piense el lector cómo podemos interpretar una afirmación en la que cuantificamos sobre todos los conjuntos de todas las jerarquías. La semántica de teoría de conjuntos usual explica las condiciones de verdad de los cuantificadores haciendo uso de conjuntos que constituyen los dominios de las variables cuantificadas. Pero el conjunto que necesitariamos ahora ( $\mathrm{y}$ al cual tendrían que pertenecer los conjuntos de todas las jerarquías) no está en la jerarquía.

\section{Las paradojas y el punto de vista del ojo de Dios}

En su artículo "Realism with a Human Face", 15 nos recuerda Putnam que "Gödel made it quite clear that he did not think the semantic paradoxes had been solved (as opposed to the set theoretic paradoxes, which he did think

15 Incluido en Putnam, Realism with a Human Face, ed. por James Conant, Harvard University Press, Cambridge, 1990. 
had been solved)" (op. cit., p. 15). Putnam se muestra de acuerdo con aquella opinión, al menos en lo que respecta a las paradojas semánticas. Una de sus reflexiones al respecto es la siguiente. Para explicar la solución clásica a esas paradojas debemos introducir una jerarquía de lenguajes, cada uno de los cuales tiene su propio predicado "es verdadero". Debe abandonarse la idea de una noción única de verdad, aplicable a cualquier lenguaje. Pero entonces surge la pregunta clave: "But in what language is Tarski himself supposed to be saying all this?" (p. 13). Claramente, "one has to stand outside the whole hierarchy even to formulate the statement that the hierarchy exists" (p. 14). Hay que adoptar "God's eye view" para explicar la solución a las paradojas semánticas.

No se puede exagerar la importancia de las observaciones anteriores. Hay problemas básicos acerca de nuestro aparato conceptual usual que no están resueltos. Tarski hizo un análisis del concepto de verdad; para que sus teorías al respecto fueran viables debió introducir la solución antes aludida de las paradojas semánticas y pareció entonces que había brindado una aclaración del concepto. Pero podemos preguntarnos si las aserciones que hace Tarski cuando expresa sus ideas sobre la jerarquía de lenguajes y temas conectados son verdaderas. Si lo son, lo son en un sentido de la noción de verdad que no solamente no está explicado por su teoría sino que parece inviable de acuerdo con ella. No podemos pensar, entonces, que los problemas acerca de la verdad que interesaron a Tarski estén resueltos. Creo que las observaciones de Putnam al respecto son profundas y creo también que dan apoyo a algunas ideas suyas sobre verdad y realismo, que no es nuestra intención exponer aquí. Pero deseo finalizar este trabajo haciendo notar que algo totalmente análogo a lo señalado por Putnam sobre las paradojas semánticas se cumple en el caso de una paradoja expuesta aquí, que no surge en la teoría de conjuntos pura, pero sí en la usual aplicación de la teoría a la semántica de $T Q$, o la teoría clásica de modelos. Si las secciones anteriores de este trabajo han dejado ver que la solución más inmediata de esa paradoja presenta dificultades similares a las del enfoque clásico de las paradojas semánticas, estas páginas habrán cumplido su objetivo principal. ${ }^{16}$

16 En "A Paradox in The Semantics of Set Theory" expongo también otra solución radicalmente distinta. Hilary Putnam la encuentra más atractiva que la solución basada en la jerarquía de teorías de conjuntos, y también yo la encuentro más prometedora, pero mis inquietudes acerca de todo el problema subsisten aún. El lector podría pensar que una teoría de conjuntos que acepte una clase universal, como NF de Quine, puede dar una solución a la paradoja. Esta propuesta fue hecha por Carlos Alchourrón en el trabajo ya citado, pero si bien $N F$ puede proporcionar el dominio para su interpretación, no puede dar la clase de pares para construir el conjunto que se asigne al ' $\epsilon$ '; después de unas conferencias que di sobre estos temas en la Universidad de Nuevo México, Estados Unidos, Bill Hart me señaló, en correspondencia, que éste es el caso, si $N F$ es consistente. Tampoco por ese lado ha llegado la tranquilidad. 